\title{
Estilos de aprendizagem dos formandos dos cursos técnicos de informática do Cefet-MG
}

\author{
Marcos Prado Amaral \\ Centro Federal de Educação Tecnológica de Minas Gerais \\ Ismar Frango Silveira \\ Universidade Cruzeiro do Sul
}

\section{Resumo}

Utilizando o instrumento N-ILS, desenvolvido e validado por Vieira Jr (2012), este artigo apresenta um mapeamento dos estilos de aprendizagem de 44 alunos do último ano do curso técnico de informática do Cefet-MG em 2014, levantando um estilo dominante e propondo a formação de oito grupos de trabalhos com 5 alunos de perfis opostos e um grupo com 4 alunos. 0 perfil dominante apurado é levemente reflexivo, moderadamente visual, leve a moderadamente sequencial e fortemente sensorial. Constatou-se que este perfil se encaixa ao curso estudado. Isto traz benefícios para os alunos, professores, escola e empresas, que receberão profissionais que gostam de fazer o que aprenderam.

Palavras-chave: Estilos de aprendizagem. Diferenças individuais. Ensino. Ensino técnico-profissional. 


\section{Learning styles of students graduating from technical courses in computer science at Cefet-MG}

Using the N-ILS instrument, developed and validated by Vieira Jr. (2012)), this article presents a survey of the learning styles of 44 last-year students of the technical course in computer science at Cefet-MG, in 2014, revealing a dominant style. A proposal was made to form 8 workgroups, with 5 students each, and one workgroup with 4 students, each group containing students with opposite styles The dominant profile found is lightly reflexive, moderately visual, lightly to moderately sequential and strongly sensory. This profile was found to fit the course. This benefits students, teachers, schools and companies, who will receive professionals who like to do what they were trained to do.

Keywords: Learning styles. Individual differences. Teaching. Professional technical training.

\section{Estilos de aprendizaje de los estudiantes del técnico en informática del Cefet-MG}

Utilizando el instrumento N-ILS desarrollado y validado por Vieira Jr. (2012), este artículo presenta un mapeo de los estilos de aprendizaje de 44 alumnos del último año del curso técnico en informática del Cefet-MG en 2014, levantando un estilo dominante y proponiendo la formación de 8 grupos de trabajos con 5 alumnos con perfiles que se oponen y un grupo con 4 alumnos. El perfil dominante calculado es ligeramente reflexivo, moderadamente visual, ligera a moderadamente secuencial y fuertemente sensorial. Se encontró que este perfil se ajusta al curso estudiado. Esto es beneficioso para los estudiantes, los profesores, la escuela y para las empresas que recibirán a estos estudiantes, con la certeza de que les gusta hacer lo que aprendieron.

Palabras-clave: Estilo de aprendizaje. Diferencias individuales. Enseñanza. Educación técnica profesional. 


\section{Introdução}

Na obtenção de seu aprendizado, cada indivíduo tem um método próprio de tratar informações. Cathólico e Oliveira Neto (2008), corroborando essa ideia, dizem que cada pessoa é única, com sua própria característica, habilidade, preferência, maneira de pensar e agir. Esses autores definem tais maneiras, particulares, de perceber e trabalhar as informações, como "Estilos de Aprendizagem". Oliveira (1998) afirma que não existe aprendizagem, mas indivíduos que aprendem com toda sua idiossincrasia e no seu contexto particular.

Pennings e Span (1991) enfatizam que os estilos de aprendizagem estão relacionados com a forma com que um indivíduo pensa, sabe, percebe, lembra, aprende ou decide.

Para Lopes (2002), os estilos de aprendizagem influenciam o processo de obtenção do conhecimento. Estes são extremamente complexos, não se restringindo apenas à aquisição de respostas, ou mesmo de conhecimentos, mas envolvendo inúmeras variáveis que se combinam de diferentes formas e estão sujeitas à influência de fatores externos, internos, individuais e sociais.

Freitas, Dornellas e Belhot (2006) descrevem que Felder e Brent (2005), Kolb (1984) e Keirsey e Bates (1984) têm em comum a ideia de que os alunos devem ter, à sua disposição, os elementos que os conduzam aos melhores resultados de aprendizagem e que supram as exigências a que estarão sujeitos, em suas atividades profissionais futuras, levando-os a uma mudança em suas perspectivas individuais (motivação, atitude e comportamento) e perspectivas profissionais (recursos e competências essenciais). E o professor tem um função importante neste cenário, dentro de seu papel mediador importante na aprendizagem. E sua função, segundo Alliprandini, Pullin e Guimaraes (2011), deve ser a de transferir, progressivamente, aos alunos o controle de sua aprendizagem. Por isso, suas ações devem promover a autonomia dos alunos na aprendizagem, sejam relacionadas ao seu desenvolvimento pessoal, sejam à sua futura capacitação profissional, preparando-os para enfrentar os desafios do mercado de trabalho. Este é fator importante na formação de alunos dos cursos técnicos, pois, apesar de, normalmente, se formarem entre os 17 e 19 anos, são cobrados a agir como se já tivessem mais maturidade profissional. São estes os alunos que foram objetos desta pesquisa.

Este paradigma, ainda, é afetado pela evolução tecnológica atual. Esta evolução tem forçado mudanças profundas no processo de ensino e aprendizagem, tornando-os mais dinâmicos e derrubando as barreiras do tempo e do espaço. De acordo com Barros e Amaral (2007), delinear os estilos de aprendizagem, mais que a necessidade de se conhecer a forma de aprender do ser humano, tem a função 
de ajudar na adaptação aos processos de mudanças advindos da tecnologia, que flexibilizam as formas e os conteúdos da aprendizagem. E reforçam o novo papel a ser desempenhado pelo professor.

Desta maneira, seria importante o conhecimento prévio dos diferentes estilos de aprendizagem dos alunos, para que o professor pudesse adequar o seu modo de ensinar a eles, tornando as disciplinas mais prazerosas de serem estudadas, levando a um maior nível de aproveitamento do aluno, tanto quanto maiores forem os patamares de aprendizagem alcançados.

Baseado nesta nova realidade é que este trabalho foi proposto, mapeando os estilos de aprendizagem dos alunos do último ano do curso técnico de informática do Centro Federal de Educação Tecnologia de Minas Gerais (Cefet-MG) tendo como base o ano de 2014.

\section{Objetivo}

A necessidade de aperfeiçoar o processo educacional torna o conhecimento dos estilos de aprendizagem, segundo Valente, Abib e Kusnik (2007), um elemento importante, levando o seu conceito a ser uma das variáveis críticas presentes no processo educacional, permitindo aos professores refletir sobre a melhor maneira de ensinar.

Além disso, cursos técnicos têm peculiaridades específicas, pois são compostos de dois núcleos: o geral, em que são ministradas as disciplinas do $2 .^{\circ} \mathrm{grau}$, e 0 técnico, no qual são ministradas as disciplinas de formação técnica, levando a uma carga horária pesada e cansativa para os alunos. Isto deveria levar os professores a procurarem maneiras diferentes de ministrar seus conteúdos.

Para ajudar os professores nesta tarefa, é que este levantamento foi feito, procurando descobrir qual o estilo de aprendizagem predominante entre os alunos do Curso Técnico de Informática do Cefet-MG e se, também, este estilo estaria de acordo com o perfil de um técnico em informática.

Mediante este levantamento, foi proposta, ainda, a formação de grupos de TCC (Trabalho de Conclusão do Curso) com alunos de perfis de aprendizagem diferentes. O TCC é uma etapa pedagógica do último do curso de informática analisado, em que os alunos são reunidos em grupos de no máximo 5 alunos, com o intuito de desenvolverem um trabalho que tenha uma aplicação prática, baseado nos conceitos que foram ensinados durante todo o curso e tendo em vista a formação profissional esperada: 
A formação profissional do egresso do curso técnico em informática do Cefet-MG provêthe autonomia suficiente para exercer atividades relacionadas a hardware e softwares, no desenvolvimento de projetos, instalações, produção e manutenção de sistemas informatizados, bem como a capacidade de supervisionar equipes de trabalho que venham a desenvolver essas atividades, atuando sobre o próprio meio, refletindo e agindo, criando e inovando. Tem em seu mercado de trabalho empresas e organizações industriais, comerciais ou de prestação de serviços, públicas e privadas, além de poder atuar como autônomo". ${ }^{1}$

O TCC se inicia conjuntamente com o ano letivo, terminando também com ele, etapa na qual há uma apresentação a uma banca examinadora, que o julga. Esperase, com esta formação, proporcionar aos alunos a convivência com a diversidade que possa levá-los ao desenvolvimento de seu pontos fracos, resultando em maior riqueza pessoal e profissional.

Assim, especificamente, este trabalho propõe a formação desses grupos de TCCs de forma heterogênea, compostos por alunos de perfil opostos, permitindo às pessoas que apresentem diversidades trabalhar em conjunto, pois, segundo Lopes (2002), não é só a mediação do professor que provoca modificações no desenvolvimento dos alunos, mas a interação dos alunos entre si. Com grupo de alunos heterogêneos, aqueles mais adiantados poderão contribuir substancialmente para o desenvolvimento dos outros.

Para o levantamento dos estilos de aprendizagem, foi usado o questionário $\mathrm{N}$-ILS, chamado de novo ILS e desenvolvido por Vieira Jr (2012). Este é derivado do Índice de Estilos de Aprendizagem (Index of Learning Styles - ILS), desenvolvido por Felder e Soloman (1991).

Ele foi disponibilizado para alunos, de forma on-line, por meio do Google Docs: <https://docs.google.com/forms/d/1lgGR843EKvTrvkpXTLVERHBGfT30q1fD0uJlx yibxh0/viewform? usp=send_form>. A escolha deste instrumento se deu, por ser ele derivado do ILS de Felder e Silverman (1998), que, segundo os autores, foi desenvolvido para ser aplicado com alunos da área de engenharia, que é a área dos cursos em questão.

\section{Estilos de aprendizagem}

Garcia Cué, Rincon e Garcia (2009), citando Alonso et al. (1994), Garger Guild (1998), Montar e Rayner (1999) e Lozano (2000) descreveram que estilos podem ser definidos como um conjunto de habilidades, preferências, tendências e atitudes

1. Disponível em: < http://www.decom.cefetmg.br/site/tec_informatica/apresentacao.html >. Acesso em: 19 jul. 2014) 
que tem uma pessoa para fazer alguma coisa e que se manifesta por um padrão de comportamento e habilidades diferentes que a distinguem de outras pessoas, sob um único rótulo de como você dirige, veste-se, fala, pensa, aprende, conhece e é ensinado.

Estilos de aprendizagem, de acordo com Alonso e Gallego (2002), são recursos cognitivos, afetivos e fisiológicos, que servem como indicadores, relativamente estáveis, de como os alunos percebem, interagem e respondem a seus ambientes de aprendizagem.

Barros e Amaral (2007) afirmam que os estilos de aprendizagem referemse a preferências e tendências altamente individualizadas de uma pessoa, que influenciam sua maneira de aprender um conteúdo.

Para Lopes (2002), os estilos de aprendizagem são considerados uma dimensão bipolar e se referem a um modo preferencial de abordar o conteúdo da aprendizagem. E que não são bons ou ruins, apenas expressam uma tendência.

Pennings e Span (1991) apresentam algumas definições interessantes sobre estilos de aprendizagem, relacionando-as com seus atores:

(i) forma como os indivíduos respondem a estímulos ambientais, emocionais, sociológicos e físicos; (Dunn \& Dunn, 1978)

(ii) orientações de aprendizagem, mais precisamente, a consistência na abordagem de tarefas de aprendizagem; (Entewistle, 1988)

(iii) reação estratégica adaptativa para uma situação de aprendizagem particular, cuja intensidade depende, igualmente, de fatores como o nível de interesse e de ansiedade e com estilos estáveis vinculados às características de personalidade e motivação; (Ford , 1981)

(iv) dimensão da personalidade, relacionados à forma particular de adquirir conhecimentos, habilidades ou atitudes mediante a experiência ou anos de estudos, que seriam um subconjunto dos estilos cognitivos; (Sadler-Smith, 1998)

(v) predisposição do aluno em adotar uma estratégia particular de aprendizagem independentemente das exigências das tarefas; (Schmeck, 1983)

De acordo com Carvalho e Novo (2005), os estilos de aprendizagem estão relacionados ao modo como as pessoas captam as informações e, por conseguinte, o seu aprendizado. Para esses autores, a captação do aprendizado se dá por meio dos sentidos, da visão, da audição, do tato, do olfato, do paladar e quanto maior o número de sentidos utilizados no processo de aprendizagem, maior será a probabilidade de fixação do conteúdo. São esses sentidos que formam o estilo de aprendizagem de cada ser humano. 
Lopes (2002), em seu trabalho, cita que Fuhrman e Grasha (1983) consideram os estilos de aprendizagem como uma interação social e que seria interessante aos processos de ensino-aprendizagem englobar o conteúdo, o aluno, o professor e a escola; nos quais professor e aluno necessitam discutir e experimentar formas alternativas de ensinar e aprender - é importante que esta discussão seja apoiada e incentivada pela escola. Na mesma direção, Cathólico e Oliveira Neto (2008) afirmam que um conhecimento mais confiável dos estilos de aprendizagem dos alunos permite compreender as formas como eles preferem aprender. E que uma maneira de se conhecer estilos de aprendizagem é mapeá-los mediante instrumentos padrões. Ainda, segundo os autores, de posse dos perfis mapeados dos alunos, podem-se desenvolver novas formas de ensino, que poderão atingir suas diferentes necessidades de aprendizagem.

Corroborando este paradigma, Hoehstein (2011) descreve que vários autores, em suas pesquisas de mapeamento de como as pessoas recebem e processam as informações para adquirir conhecimentos, desenvolveram modelos, para ajudar no levantamento dos estilos de aprendizagem, e que os mais utilizados na literatura são: Felder-Silverman (1991), Bloom (1972), Kolb (1984), Ford-Chen (2001) e Inteligências Múltiplas de Gardner (1994). Esses modelos foram usados para desenvolver vários instrumentos, que permitem um mapeamento confiável dos estilos de aprendizado. Os instrumentos mais comuns são os inventários, na forma de questionários, que são baseados em estilos de aprendizagem predefinidos.

Neste trabalho, foi usado o questionário desenvolvido por Vieira Jr (2012), que é baseado na teoria de Felder-Silverman (1991).

\section{Novo Índice de Estilos de Aprendizagem (N-ILS)}

Como já foi dito, existem instrumentos de diagnóstico para se conhecerem os estilos de aprendizagem, e os mais usuais são os questionários, ou testes. Estes podem ser aplicados em diversas situações de aprendizagem, independentemente da área ou conteúdo a ser desenvolvido.

Vários autores têm apresentado, segundo Garcia Cué, Rincon e Garcia (2009), instrumentos de diagnóstico ao longo dos anos em vários campos de pesquisa educacional, empresarial, psicológica e pedagógica e deram origem a um grande número de livros e publicação de artigos científicos. Alguns deles são mostrados no quadro 1. 
QUADRO 1: Instrumentos de diagnósticos

\begin{tabular}{c|c}
\hline Autores & Instrumentos \\
\hline $\begin{array}{c}\text { Alonso, Gallego e Honey } \\
(1992,1994)\end{array}$ & $\begin{array}{c}\text { Questionário Honey-Alonso de Estilos de } \\
\text { Aprendizagem (CHAEA) }\end{array}$ \\
\hline Felder e Soloman (1991) & Índice de Estilos de Aprendizagem (ILS) \\
\hline David Kolb (1976) & Inventário de Estilos de Aprendizagem \\
\hline James Keefe (1979) & Perfil de Estilos de Aprendizagem \\
\hline Honey e Mumford (1988) & Questionário de Estilos de Aprendizagem \\
\hline S. Whiteley e K. Whiteley (2003) & $\begin{array}{c}\text { Inventário de Estilos de Aprendizagem do } \\
\text { Projeto Memletics }\end{array}$ \\
\hline
\end{tabular}

Fonte: Adaptado de Garcia Cué, Rincon e Garcia, 2009, p.3

0 instrumento utilizado neste trabalho foi o $\mathrm{N}$-ILS, chamado de novo ILS e desenvolvido, testado e validado por Vieira Jr (2012)². Ele é uma derivação do Índice de Estilos de Aprendizagem (Index of Learning Styles - ILS), desenvolvido por Felder e Soloman (1991).

Não foi usado o questionário de Felder e Soloman (1991), pois, na sua versão em português, ele apresenta inconsistências que foram mostradas pelo próprio Vieira Jr (2012) e também por Lopes (2002). O N-ILS, como derivação do ILS de Felder e Silverman (1998), segue toda a sua base teórica.

Segundo Felder et al. (2007), o Índice de Estilos de Aprendizagem (ILS), criado por Felder e Soloman, foi concebido para avaliar preferências de aprendizagem em quatro dimensões bipolares, sendo baseado no modelo de estilo de aprendizagem formulado por Felder e Silverman. Felder e Spurlin (2005) descrevem, resumidamente, as quatro dimensões como se segue:

- Ativo/Reflexivo: Aprendizes Ativos tendem a ter a facilidade de reter e compreender melhor a informação quando participam ativamente de atividades, discutindo, aplicando ou explicando para outros. Gostam de trabalhar em grupos. Os Aprendizes Reflexivos gostam de refletir primeiramente sobre a informação. Assim, tendem a ser mais lentos para iniciar uma atividade. Preferem trabalhar individualmente.

2. A validação pode ser conferida na tese de doutorado de Vieira Jr (2012) e não faz parte do escopo deste trabalho. 
- Visual/Verbal: Aprendizes Visuais aprendem mais facilmente por meio de gráficos, mapas, diagramas, imagens e experiências. Se a informação é apresentada de uma forma escrita ou verbal, eles provavelmente esquecerão rapidamente. Já os Aprendizes Visuais aprendem melhor as informações faladas ou escritas.

- Sequencial/Global: Aprendizes Sequenciais gostam de aprender a atividade de forma parcial. Ganham entendimento em passos lineares, cada um derivado do anterior. Normalmente seguem caminhos lógicos e graduais na solução de um problema. Têm facilidade para explicar aos colegas etapas do serviço a ser executado. Os Aprendizes Globais preferem aprender com grandes saltos, absorvendo a informação no todo. Têm facilidade de juntar elementos de maneira nova. Podem ter dificuldade de explicar como chegaram à solução de um problema. Seu foco está na síntese e no pensamento sistêmico.

- Sensorial/Intuitivo: Aprendizes Detalhistas-Sensoriais têm facilidade de resolver problemas mediante procedimentos bem estabelecidos, sendo pacientes com detalhes, apreciando trabalhos de manipulação, experimentais e repetitivos. Os Aprendizes Generalistas-Intuitivos preferem descobrir possibilidades, apreciando as inovações e não se sentem bem com exercícios repetitivos. Sentemse confortáveis com abstrações e são rápidos e criativos.

0 N-ILS, de acordo com Vieira Jr (2012), é composto por 20 questões, em que cada uma das quatro dimensões bipolares possui 5 perguntas relacionadas a ela. 0 cálculo das preferências é feito como no questionário de Felder e Soloman (1991). Cada dimensão apresenta duas opções de respostas (modelo de item denominado - escolha forçada). É solicitado ao participante que marque um "X" na letra "a" ou "b" para indicar sua resposta a cada uma das questões. Desta maneira a dimensão bipolar pode ter uma nota que varia de 1 a 11.0 nível de predominância de estilo indicado por pontuações de 1 a 3 na escala indica "leve" preferência entre ambas dimensões; pontuações de 5 a 7 na escala indicam preferência "moderada" por uma das dimensões; e a pontuação de 9 a 11 na escala indica "forte" preferência por uma das dimensões.

Baseadas nessas primícias é que foram colocadas as três questões gerais para a pesquisa aqui feita: "Quais os estilos de aprendizagem dos alunos do último ano do curso técnico em informática do Cefet-MG?", "Qual é o perfil dominante apurado?", "Este perfil está de acordo com o curso técnico em informática?"

\section{Metodologia}

A pesquisa desenvolvida pode ser caracterizada como teórico-empírica, sendo composta da aplicação do questionário ILS desenvolvido por Felder e Soloman (1991), 
com o intuito de identificar e analisar os estilos de aprendizagem dos discentes do último ano do curso técnico de informática, do Cefet-MG, na sua forma integrada. Os dados foram coletados por meio de questionários preenchidos pelos alunos, durante o período de março a abril de 2014. Os questionários foram disponibilizados, de forma on-line, por meio do Google Docs.

Além dos questionários, os links trazem explicações sobre a pesquisa, definições sobre estilos de aprendizagem e sobre o ILS. Em outro link é solicitada a aprovação dos responsáveis pelos alunos, caso sejam eles menores de idade.

Dos 49 alunos que compõem o $3 .^{\circ}$ anos do curso de informática, 89,79\% responderam ao questionário ${ }^{3}$, ou seja 44 alunos.

Depois de preenchidos, os questionários foram transcritos em planilha eletrônica do Excel ${ }^{\circledR}$ para tabulação e tratamento dos dados. A apresentação e análise dos resultados foram realizadas com o auxílio de recursos da estatística descritiva. Foi enviado um e-mail a cada participante com o seu perfil apurado.

Os alunos da amostragem têm idade entre 16 e 19 anos, em que $28,6 \%$ tem 16 anos, $45,7 \%$ tem 17 anos, $20 \%$ tem 18 anos e 5,7\% 19 anos. Um dado interessante é que $28,6 \%$ são alunas.

A pesquisa feita é um estudo de caso, pois estuda um conjunto de alunos com o objetivo de compreender seus estilos de aprendizagem. Essa ideia é corroborada por Goldenberg (2004) ao afirmar que o estudo de caso não é uma técnica específica, mas uma análise holística, a mais completa possível, que considera a unidade social estudada como um todo: seja um indivíduo, uma família, uma instituição, seja uma comunidade, com o objetivo de compreendê-los em seus próprios termos. No estudo de caso, segundo Ludke e André (1986), o pesquisador procura revelar a multiplicidade de dimensões, presentes numa determinada situação ou problema pesquisado, focalizando-o como um todo. Assim, nesse ponto, a pesquisa é qualitativa, pois busca, nos estilos levantados, manifestações e observações livres a respeito do perfil dominante com relação ao curso tomado como parâmetro.

As abordagens qualitativas, segundo Günther (2006), que tendem a ser associadas a estudos de caso, dependem de estudos quantitativos, que visem gerar resultados generalizáveis, isto é, parâmetros. E, num estudo de caso, é possível utilizar tanto procedimentos qualitativos quanto quantitativos. Assim, esta pesquisa é também quantitativa, pois quantifica os dados para levantamento de um perfil dominante.

A pesquisa é, também, um levantamento, pois a amostra foi coletada por questionários aplicados via Internet. 0 levantamento, para Gil (2002), envolve a interrogação direta das pessoas, cujo comportamento se deseja conhecer. Nesse caso,

3. Disponível em: <https://docs.google.com/forms/d/1lgGR843EKvTrvkpXTLVERHBGfT30q1fDOuJlxyibxh0/ viewform?usp=send_form>. Acesso em: 19 jul. 2014. 
segundo Cathólico e Oliveira Neto (2008), procede-se a solicitação de informações a um grupo significativo de pessoas, acerca do problema estudado para, em seguida, mediante análise quantitativa, obter conclusões correspondentes aos dados coletados.

As etapas da pesquisa são: definição da amostra, recebimento dos dados e respectiva avaliação, tabulação, análise e conclusão.

\section{Análise dos resultados}

Foi realizada uma análise quantitativa dos 44 questionários respondidos, com a verificação dos números absolutos e percentuais. Identificaram-se os estilos predominantes e levantaram-se variações encontradas nos estilos de cada um, classificados pelo próprio questionário em leve, moderado ou forte. Esses resultados estão mostrados na figura 1, com as nomenclaturas: Ativo/Reflexivo, Visual/Verbal, Sequencial/Global, Detalhista/Intuitivo, nas modalidades: Leve (L), Moderado (M), Forte (F).

FIGURA 1: Visualização gráfica dos estilos de aprendizagem dos alunos

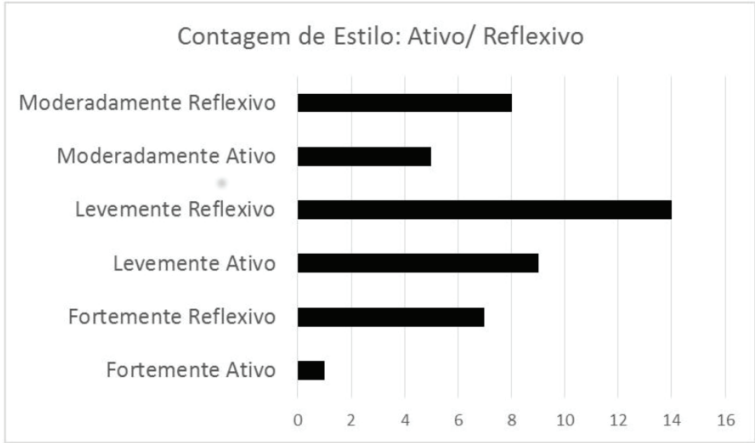

Contagem de Estilo: Sequencial/ Global

Moderadamente Sequencial

Moderadamente Globa

Levemente Sequencial

Levemente Global

Fortemente Sequencial

Fortemente Globa
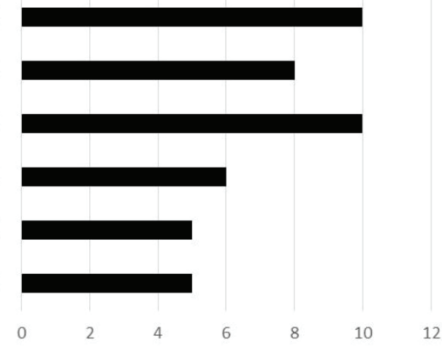

Contagem de Estilo: Visual/ Verbal

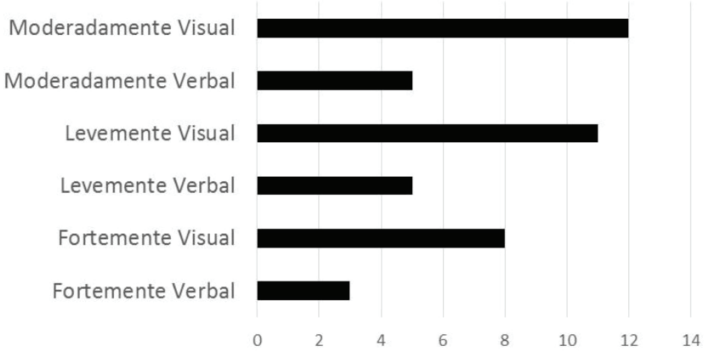

Contagem de Estilo: Sensorial/ Intuitivo

Moderadamente Sensorial

Moderadamente Intuitivo

Levemente Sensorial

Levemente Intuitivo

Fortemente Sensorial

Fortemente Intuitivo

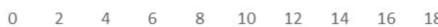

Fonte: Microsoft Excel® 2013 
A dimensão bipolar Ativo/Reflexivo 15/29 (34\%/66\%) demostra uma propensão para alunos reflexivos, que preferem refletir primeiramente sobre a informação e preferem trabalhar individualmente. Esse perfil condiz com o curso do qual os alunos participam, pois é o perfil próprio para quem vai atuar no desenvolvimento de projetos, instalações, produção e manutenção de sistemas informatizados 4 . Ao ministrar suas disciplinas, os professores devem dar a estes alunos todas as informações necessárias para os problemas propostos. Depois, esperar que eles apresentem suas soluções, para então analisar, individualmente, cada uma das soluções, mostrando o que está bom e o que pode ser melhorado. Aqui os professores só devem ter cuidado no momento de passar as informações para os alunos, devendo cobrar deles de acordo com essas informações.

A dimensão Visual/Verbal 31/13 (70\%/30\%) mostra que a maioria expressiva dos alunos têm facilidade de aprender, quando são ensinados por meio de gráficos, quadros, figuras, fluxogramas - perfil muito bom para quem vai atuar com informática. Sua aprendizagem deve ser bastante visual, com os alunos manipulando telas, gráficos, fluxogramas e esquemas em blocos. Nas proposições de aulas, é recomendável fornecer farta bibliografia e, se possível, criar vídeos sobre o conteúdo.

A dimensão Sequencial/Global 25/19 (57\%/43\%) mostra que os alunos gostam de aprender as atividades uma etapa após a outra, ganhando entendimento em passos lineares, cada um derivado do anterior. Normalmente seguem caminhos lógicos e graduais na solução de um problema. Este perfil é o desejado para quem vai atuar com desenvolvimento de sistemas informatizados. Devem ser ensinados a como trabalhar com estruturas de programação. Aprendem melhor com a proposição, bem detalhada, de trabalhos de desenvolvimento de softwares. Ao incentivá-los a trabalhar em equipe, deve-se ter o cuidado de dividir bem as tarefas, em que cada um fica responsável por uma parte funcional de um software, para depois se reunirem e, um por um, irem explicando para os colegas da equipe o que desenvolveram e até como foi desenvolvido.

A dimensão Sensorial/Intuitivo 34/10 (77\%/23\%) mostra que a maioria considerável dos alunos gosta de receber as informações por meio de procedimentos bem estabelecidos, apreciando o trabalho metódico - perfil próprio de quem vai atuar com desenvolvimento e manutenção de projetos informatizados. Eles têm mais facilidade de aprender, quando thes são passados problemas que tenham conexão com o mundo real e que envolvem manipulação e experimentação, como as práticas de laboratório. Ao ensinar-lhes conceitos e procedimentos, deve-se

4. Disponível em: <http://www.decom.cefetmg.br/site/tec_informatica/apresentacao.html>. Acesso em: 19 jul. 2014. 
dar exemplos de como esses conceitos se aplicam na prática.

Pela análise dos gráficos na figura 1, determina-se, quantitativamente, que o estilo de aprendizagem dominante, entre os alunos, é levemente reflexivo, moderadamente visual, leve a moderadamente sequencial e fortemente sensorial. Estilo este que, conforme analisado qualitativamente, está de acordo com a área de atuação do técnico em informática.

Na definição dos grupos de TCC, baseada em seus estilos de aprendizagem, foi usada a denominação genérica de aluno, seguida por número sequencial, obedecendo à ordem alfabética dos nomes. Essa ação tem o intuito de preservar o anonimato dos entrevistados.

Com os perfis em mãos, passou-se para a divisão dos grupos de TCC, em 8 grupos de 5 alunos e 1 grupo de 4 alunos (44 alunos). A composição dos grupos obedeceu ao seguinte ranqueamento definido pelo pesquisador: (i) grupos formados pela sequência de um aluno sequencial, um aluno global, um aluno visual, um aluno verbal e um aluno intuitivo, num total de 3 grupos; (ii) grupos formados pela sequência de um aluno sensorial, um aluno intuitivo, um aluno visual, um aluno verbal e um aluno sequencial, num total de 3 grupos; (iii) grupos formados pela sequência de um aluno sequencial, um aluno global, um aluno intuitivo, um aluno sensorial, um aluno visual, num total de 2 grupos; (iv) um aluno sequencial, um aluno global, um aluno visual, um aluno verbal, num total de 1 grupo.

\section{Considerações finais}

É fato que os processos de aprendizagem desempenham um papel central no desenvolvimento do aspecto cognitivo, emocional e social de um aluno. Por isso é fundamental conhecer os estilos de aprendizagem de cada aluno para compreender como eles se relacionam com o conhecimento e como é sua forma de aprender.

Reconhecer os vários perfis existentes e construir didáticas baseadas nesses perfis pode levar os alunos a uma maior satisfação, autonomia e aproveitamento nos estudos.

A finalidade deste trabalho foi a identificação dos estilos de aprendizagem dos alunos do último ano do curso técnico de informática do Cefet-MG mediante análise de suas respostas ao Novo Índice de Estilos de Aprendizagem (N-ILS), preenchido de forma on-line.

Com esta análise foi possível confrontar os estilos de aprendizagem dos alunos e definir um estilo desejado para alunos do curso que eles frequentam. Como resposta, pode-se afirmar que os 44 entrevistados têm o estilo desejado para o 
curso. E que o estilo dominante para esses aprendizes deve-se aproximar de um levemente reflexivo, moderadamente visual, leve a moderadamente sequencial e fortemente sensorial. Esta informação pode ser trabalhada pela escola, para melhor focar as ementas das disciplinas, e pelos professores, para direcionar seus materiais de ensino e suas aulas. As empresas, também, se beneficiam, sabendo que terão profissionais que fazem o que gostam e têm a facilidade de trabalhar com o que aprenderam.

Foram definidos, ainda, baseados neste levantamento, 8 grupos de TCC com 5 alunos e 1 grupo de 4 alunos, englobando os 44 alunos. Os grupos foram compostos de acordo com o ranqueamento definido pelo pesquisador, de forma e serem heterogêneos em seus estilos de aprendizagem. Com esta composição, o pesquisador espera proporcionar aos alunos um convívio das diferenças. E esta pode ser considerada uma experiência interessante, pois não é comum as escolas, de qualquer nível, se atentarem para este tipo de detalhe.

Dado o espaço amostral, pode-se dizer, também, que não é possível assumir a generalização dos resultados. Como esse trabalho é inédito no âmbito do curso de informática do Cefet-MG, pretende-se, como trabalhos futuros, aplicá-lo nos próximos anos, para estender essa análise.

A aplicação do Novo Índice de Estilos de Aprendizagem (N-ILS), para identificar os estilos de aprendizagem com vista a melhorar a motivação dos alunos às aulas, é mostrada por Vieira Jr. (2012) como confiável e válida. A aplicação desse índice, dentro dos parâmetros determinados para essa pesquisa, se feita no começo do ano letivo, pode ajudar a construir grupos de trabalhos com perfil heterogêneo. Com isto, pode-se formar grupos de trabalhos de conclusão de curso (TCC), de forma heterogênea, de maneira a permitir o convívio de pessoas que apresentem diversidades para trabalhar em conjunto, permitindo a experiência dessa convivência (com a diversidade), com o desenvolvimento de pontos fracos e fortes dos alunos, preparando-os melhor para os cenários da vida real, resultando em maior riqueza pessoal e profissional.

\section{Referências}

ALONSO, Catalina María; GALLEGO, Domingo José; HONEY, Peter. Los estilos de aprendizaje: procedimientos de diagnóstico y mejora. Madrid: Mensajero, 2002.

ALLIPRANDINI, Paula Maiza Zedu; PULLIN, Elsa Maria Mendes Pessoa; GUIMARÃES, Sueli Édi Rufini. Estilos de aprendizagem de alunos ingressantes em um curso de pedagogia de uma instituição pública do norte do Paraná e implicações para a prática pedagógica. Revista Iberoamericana de Educación. n. 
55/5. 15 jun. 2011.

BARROS, Daniela Melaré Vieira; AMARAL Sergio Ferreira. Estilos de aprendizagem no contexto educativo de uso das tecnologias digitais interativas. In: SIMPÓSIO INTERNACIONAL SOBRE NOVAS COMPETÊNCIAS EM TECNOLOGIAS INTERATIVAS NA EDUCAÇÃO, 1., 2007, São José dos Campos, SP, Anais... São José dos Campos, 2007.

CARVALHO, Fernanda Antoniolo Hammes de; NOVO, Magda Suzana. Aprender como Aprender: Otimização da Aprendizagem. Disponível em: <http://www.seer. furg.br/momento/article/download/618/158>. Acesso em: 26 jul. 2014 CATHÓLICO, Roberval Aparecido Rodrigues; OLIVEIRA NETO, Jose Dutra de. Inventario de estilos de aprendizagem em um curso de eletrotécnica. Revista Eletrônica de Educação e Tecnologia do SENAI-SP. v. 3, n. 6, 2009.

FELDER, Richard Martin et. al. A Study of the Reliability and Validity of the FelderSoloman Index of Learning Styles. In Engineering Education 96(4), pp. 309-319, 2007.

FELDER, Richard Martin; SILVERMAN, Linda. Learning and Teaching Styles. In Engineering Education 78(7), pp. 674-681, 1988.

FELDER, Richard Martin; SOLOMAN. Barbara. Index of learning styles questionnaire. North Carolina State University, Raleigh, 1991. Disponível em: <http://www.engr. ncsu.edu/learningstyles/ilsweb.html>. Acesso em: 26 jul. 2014.

FELDER, Richard Martin; SPURLIN, Joni. E. Applications, Reliability, and Validity of the Index of Learning Styles. International Journal Engineering Education, 21(1), pp. 103-112, 2005.

FREITAS, Alessandra Aparecida; DORNELLAS, Danielle Vasconcellos; BELHOT, Renato Vairo. Requisitos profissionais do estudante de engenharia de produção: uma visão através dos estilos de aprendizagem. GEPROS - Ano 1, n. 2, abr/2006, pp. 125-135.

GARCIA CUÉ, José Luís; RINCON, José Antonio Santizo; GARCIA, Catalina María Alonso. Uso de las TIC de acuerdo a los estilos de aprendizaje de docentes y discentes. Revista Iberoamericana de Educación. n. 48/2. 10 jan. 2009.

GIL, Antônio Carlos. Como elaborar projetos de pesquisa. 4 ed. São Paulo: Atlas S/A. 2002

GÜNTHER, Hartmut. Pesquisa Qualitativa Versus Pesquisa Quantitativa: Esta é a Questão?. Psicologia: Teoria e Pesquisa, v. 22, n.2 pp. 201-210, maio-ago/2006, Brasilia, DF.

HOEHSTEIN, Gilcimar et. al. Diagnóstico do estilo de aprendizagem do aluno a partir de ferramentas de comunicação. Cadernos de Informática: Anais do VI Congresso Ibero-americano de Telemática (CITA 2011), v. 6, n. 1, 2011, Gramada, RS. pp. 87-94. 
LOPES, Wilma Maria Guimarães. ILS - Inventário de estilos de aprendizagem de Felder- Soloman: investigação de sua validade em estudantes universitários de Belo Horizonte. 85 f. Dissertação (Mestrado) - Universidade Federal Santa Catarina, Florianópolis, 2002.

LUDKE, Menga; ANDRÉ, Marli Elisa Dalmazo Afonso de. Pesquisa em Educação: Abordagens Qualitativas. Temas Básicos de Educação e Ensino. EPU, S.P., 1986. $99 \mathrm{p}$.

OLIVEIRA, José Henrique Barros de. Cabeças bem feitas ou bem cheias? In: Revista do Colégio Internato dos Carvalhos. Vol. II, n. 1, pp.55-75, maio/1998.

PENNINGS, Albèr; SPAN, Pieter. Estilos cognitivos e estilos de aprendizagem. In: ALMEIDA, L. (Org.). Cognição e Aprendizagem Escolar. Coleção Temas de Psicologia. Porto: APPORT, 1991.

VIEIRA Jr. Neltom. Planejamento de um ambiente virtual de aprendizagem baseado em interfaces dinâmicas e uma aplicação ao estudo de potência elétrica. $233 \mathrm{f}$. Tese (Doutorado) - Universidade Estadual Paulista “Júlio Mesquita Filho”. Campus de Itha Solteira, 2012.

Recebido em outubro de 2014

Aprovado em dezembro de 2014

Marcos Prado Amaral é doutorando na Universidade Cruzeiro do Sul e professor do Centro Federal de Educação Tecnológica de Minas Gerais.. E-mail: marcaopamaraldgmail.com

Ismar Frango Silveira é doutor em realidade virtual distribuída aplicada à educação pela Universidade de São Paulo e professor da Universidade Presbiteriana Mackenzie e da Universidade Cruzeiro do Sul. E-mail: ismarfrangodagmail.com 